\title{
La aplicación intraarticular de corticoides antes del ejercicio no mostró beneficios en pacientes con artrosis de rodilla
}

\section{Intraarticular corticosteroid injection before exercise did not show benefits in patients with osteoarthritis}

of the knee

\section{Objetivos}

Evaluar la eficacia de la aplicación intraarticular de una inyección de corticoides en pacientes con artrosis de rodilla previo a un programa de ejercicios.

\section{Diseño}

Ensayo clínico aleatorizado, doble ciego y controlado con placebo.

\section{Lugar}

Hospital Universitario de Copenhague. Desde el 1 de octubre de 2012 hasta el 2 de abril de 2014.

\section{Pacientes}

Pacientes ambulatorios $\geq 40$ años de edad, con diagnóstico de artrosis (confirmación radiográfica de artrosis tibio femoral), signos clínicos de inflamación localizada en rodilla, dolor en la rodilla durante la deambulación, índice de masa corporal de 35 o menos. Se excluyeron pacientes con inyección de corticoides o participación en terapia de ejercicios en los últimos tres meses, uso reciente o actual de corticoides orales, artritis inflamatoria, antecedentes de artroplastia de rodilla, imposibilidad de realizar ejercicios, contraindicaciones para recibir inyecciones de corticoides, síndrome de dolor regional, síndrome de
Henriksen M. JAMA Intern Med. 2015;175(6):923-930 compresión radicular.

\section{Intervención}

Se utilizó la inyección intraarticular de $1 \mathrm{ml}$ de acetato de metilpresdnisolona y $4 \mathrm{ml}$ de lidocaína $1 \%$, completando $10 \mathrm{ml}$ vs inyección intraarticular de $1 \mathrm{ml}$ de solución salina isotónica y $4 \mathrm{ml}$ de lidocaína 1\% completando $10 \mathrm{ml}$. Ambos grupos realizaron el mismo programa de ejercicios comenzando dos semanas después de la aplicación.

\section{Medición de resultados principales}

Se evaluó la subescala de dolor del cuestionario de lesiones de rodilla y artrosis KOOS (en inglés, Knee injury and Osteoarthritis Outcome), con un rango de 0 a 100, mostrando mayor mejoría a mayor score. El análisis se realizó por intención de tratar.

\section{Resultados principales}

No hubo diferencias en el resultado principal (ver tabla 1) ni en los resultados secundarios (diferentes subescalas de KOOS: síntomas, funciones de la vida diaria, calidad de vida relacionada con la rodilla, función en recreación y deportes; capacidad funcional de carga de peso; fuerza muscular de isquiotibiales, etc.).

Tabla 1: Cambio en la subescala de dolor del cuestionario KOOS entre ambos grupos a la semana 14.

\begin{tabular}{c|c|c|c|c|} 
Resultado primario & $\begin{array}{c}\text { Placebo } \\
\mathbf{N}=\mathbf{5 0}\end{array}$ & $\begin{array}{c}\text { Inyección de corticoides } \\
\mathbf{N = 5 0}\end{array}$ & $\begin{array}{c}\text { Diferencia de medias } \\
\text { (IC 95\%) }\end{array}$ & P-valor \\
\hline Dolor & $14,8(1,8)$ & $13,6(1,8)$ & $1,2(-3,8 \mathrm{a} 6,2)$ & 0,64 \\
\hline
\end{tabular}

\section{Conclusión}

La inyección de corticoide intraarticular previa a la realización de actividad física no mostró beneficios en pacientes con artrosis de rodilla.

Fuente de financiamiento/ conflicto de interés de los autores: No especificado

\section{Comentario}

El tratamiento de la artrosis debe ser realista y enfocado a las preferencias de los pacientes. El manejo de la artrosis tiende a ser conservador, usando como primera línea intervenciones basadas en la actividad física, luego se adicionan tratamientos farmacológicos y kinésicos $y$, finalmente, se sugiere la cirugía. Las indicaciones deberían orientarse a disminuir el dolor, mejorar la funcionalidad y la calidad de vida, y de este modo, facilitar la autonomía de la persona y su inserción en la comunidad

Los ejercicios físicos han mostrado ser eficaces en el tratamiento del dolor en las primeras etapas de la artrosis'. Los tratamientos analgésicos y antiinflamatorios han presentado resultados pasajeros en cuanto al dolor sin mucho aval científico que sostenga su utilización. Arrol y cols, en 2004, realizaron un metanálisis que mostraba eficacia en el tratamiento con corticoides², pero la fuerza de la evidencia era limitada por basarse en trabajos pequeños, con pocos pacientes. La eficacia del condroitin sulfato, solo o combinado con glucosamina, también fue evaluada en pacientes con este problema, observándose un beneficio modera- do aunque con preocupante riesgo de sesgo por el financiamiento de la industria de los ensayos incluidos en el metanálisis ${ }^{3}$. La proloterapia se instaló en la década de 1950 como tratamiento del dolor articular, interpretando este síntoma como relacionado con la debilidad del tejido blando (capsula, ligamentos y tendones) circundante $^{4}$. Esta terapia demostró eficacia en el tratamiento de tendinopatias y ligamentopatias, y está siendo evaluada para el tratamiento de la OA. El tapping, o vendaje funcional, es una terapia simple y de bajo costo, eficaz para disminuir el dolor articular y permitir a la persona afectada realizar ejercicios de fortalecimiento de cuádriceps, o rehabilitación ${ }^{5}$.

\section{Conclusiones del comentador}

La OA es un problema multifactorial que permite un abanico terapéutico variado y adaptable a los perfiles médicos, de los pacientes y accesos sanitarios. Toda intervención debe estar orientada a ayudar al paciente respetando sus preferencias.

Denise Cytryn [ Servicio de Medicina Familiar y Comunitaria. Hospital Italiano de Buenos Aires. denise.cytryn@ hospitalitaliano.org.ar ]

Cytryn D. La aplicación intraarticular de corticoides antes del ejercicio no mostró beneficios en pacientes con artrosis de rodilla. Evid Act Pract Ambul. 2016;19(3):81. Comentado de: Henriksen M y col. Evaluation of the Benefit of Corticosteroid Injection Before Exercise Therapy in Patients With Osteoarthritis of the Knee. A Randomized Clinical Trial. JAMA Intern Med. 2015;175(6):923-930. PMID: 25822572.

\section{Referencias}

1. Juhl C y col . Impact of exercise type and dose on pain and disability in knee osteoarthritis: a systematic review and meta-regression analysis of randomized controlled trials. Arthritis Rheumatol. 2014;66(3):622-636.

2. Arroll B y col. Corticosteroid injections for osteoarthritis of the knee: meta-analysis. BMJ 2004;328:869

3. McAlindon TE y col. Glucosamine and chondroitin for treatment of osteoarthritis: a systematic quality assessment and meta-analysis. JAMA 2000;283(11) :1469-1475.

4. Ligament and Tendon Relaxation, Treated by Prolotherapy, Hackett, Hemwald, Montgomery, Hackett Hemwald Fundation, $5^{\star}$ ed. 2008.

5.Hinman RS y col. Efficacy of knee tape in the management of osteoarthritis of the knee: blinded randomised controlled trial. BMJ. 2003 Jul 19;327(7407):135. 